\title{
Dicer-Processed Small RNAs: Rules and Exceptions
}

\author{
David Langenberger ${ }^{\mathrm{a}, \mathrm{b}}$, M. Volkan Çakir ${ }^{\mathrm{b}}$, Steve Hoffmann ${ }^{\mathrm{a}, \mathrm{b}}$, Peter F. Stadler ${ }^{\mathrm{a}, \mathrm{b}, \mathrm{c}, \mathrm{d}, \mathrm{e}, \mathrm{f}, \mathrm{g}, \mathrm{g}, \mathrm{i}, \mathrm{i}}$ \\ ${ }^{a}$ LIFE, Leipzig Research Center for Civilization Diseases, University Leipzig, Philipp-Rosenthal-Strasse 27, D-04107 Leipzig, Germany \\ ${ }^{b}$ Interdisciplinary Center for Bioinformatics, University of Leipzig, Härtelstraße 16-18, D-04107, Leipzig, Germany \\ ${ }^{c}$ Bioinformatics Group, Department of Computer Science, Härtelstraße 16-18, D-04107, Leipzig, Germany \\ studla@bioinf.uni-leipzig.de \\ ${ }^{d}$ Max Planck Institute for Mathematics in the Sciences, Inselstraße 22, D-04103 Leipzig, Germany \\ ${ }^{e}$ RNomics Group, Fraunhofer Institut für Zelltherapie und Immunologie, Deutscher Platz 5e, D-04103 Leipzig, Germany \\ ${ }^{f}$ Department of Theoretical Chemistry, University of Vienna, Währingerstraße 17, A-1090 Wien, Austria \\ ${ }^{g}$ Center for non-coding RNA in Technology and Health, University of Copenhagen, Gronnegårdsvej 3, DK-1870 Frederiksberg, Denmark \\ ${ }^{h}$ Santa Fe Institute, 1399 Hyde Park Rd., Santa Fe, NM 87501, USA \\ ${ }^{i}$ Corresponding author
}

\begin{abstract}
Canonical microRNAs are excised from their hairpin-shaped precursors by Dicer. In order to find possible exceptions to this rule and to identify additional substrates for Dicer processing we re-evaluate the small RNA sequencing data of the Dicer knockdown experiment in MCF-7 cells orignally published by Friedländer et al. [Nucleic Acids Res. 40: 37-52 (2012)]. While the well-known non-Dicer mir-451 is not sufficiently expressed in these experiments, there are several additional Dicer-independent microRNAs, among them the important tumor supressor mir-663a. We recover previously described examples of non-miRNA Dicer substrates such as tRNA-Gln and several snoRNAs. Interestingly, sdRNAs derived from box C/D snoRNAs are Dicer-independent, while those derived from box H/ACA snoRNAs are often Dicer dependent. Several pol-III transcripts, in particular the vault RNAs and the great ape specific snaRs are processed by Dicer, while the small RNAs originating from Y RNAs seem to be Dicer independent.
\end{abstract}

Keywords: sdRNAs, snoRNAs, Y RNAs, vault RNAs, snaRs 


\section{Introduction}

Canonical microRNAs are processed from a primary pol II transcript by means of the Drosha-dependent microprocessor complex (Gregory et al., 2004), resulting in a characteristic hairpin of length 60-120 nucleotides. This pre-microRNA is then transported by Exportin- 5 to the cytoplasm (Lund et al., 2004), where the hairpin is cut by Dicer into a double stranded RNA about 22nt in length with a 2nt 3'-overhang (Murchison and Hannon, 2004). Several alternative pathways that bypass Drosha have been reported. The most prominent example are mirtrons (Okamura et al., 2007; Ruby et al., 2007), whose precursor hairpins are produced by splicing. A related, mirtron-like source of small RNAs requires both splicing and exosome-mediated trimming to extract the pre-microRNA hairpin (Flynt et al., 2010; Chong et al., 2010). More recently, it was shown that a few microRNAs, in particular mir-451, are matured without the help of Dicer (Cheloufi et al., 2010; Cifuentes et al., 2010). For a recent review of the many alternative pathways for the biogenesis of microRNAs and other, microRNA-like small RNA species see e.g. (Yang and Lai, 2011).

Apparently, Dicer is not only involved in microRNA biogenesis, but appears to be involved also in the processing of other small RNA species. Short, microRNAlike RNAs are processed from a diverse set of usually well-structured non-coding RNAs that includes tRNAs (Lee et al., 2009; Cole et al., 2009; Haussecker et al., 2010; Findeiß et al., 2011; Sobala and Hutvagner, 2011), snoRNAs (Kawaji et al., 2008; Taft et al., 2009; Langenberger et al., 2010; Brameier et al., 2011), vault RNAs (Stadler et al., 2009; Persson et al., 2009), Y RNAs (Langenberger et al., 2010; Meiri et al., 2010; Verhagen and Pruijn, 2011), and snRNAs (Langenberger et al., 2010). Not much is known about the maturation of most of these small RNAs. The importance of Dicer for these small RNAs has been demonstrated in only a few cases: the small RNAs derived from human tRNA(Gln) are dependent on Dicer both in vivo and in vitro (Cole et al., 2009), see also (Babiarz et al., 2008). Some snoRNA derived sdRNAs show altered expression in mouse Dicer1 and Dgcr8 mutants (Taft et al., 2009), and processing of ACA45 derived sdRNAs requires Dicer activity but not Drosha/DGCR8 (Ender et al., 2008). Endogenous siRNAs resulting from Dicer cleavage of long hairpins, typically deriving from SINEs with tandem inverted repeat structure have been reported in (Babiarz et al., 2008).

Here we reevaluate a previously published set of RNA sequencing data (GSE31069) that compare the expres- sion of small, microRNA-sized RNAs before and after Dicer knock-down in a MCF-7 cell line (Friedländer et al., 2012). Our analysis focusses on the identification in particular of microRNAs that fail to respond to the depletion of Dicer, and conversely on those loci that are strongly Dicer-dependent but are not classified as microRNAs.

\section{Methods}

\subsection{Data and Mapping}

We downloaded a previously published sequencing data set series (GSE31069, (Friedländer et al., 2012)) from the Gene Expression Omnibus (GEO) database (Edgar et al., 2002). The data consists of four different samples, two containing short reads from the total cell content and two containing reads from the cytoplasmic fraction only. Both pairs contrast small RNA expression before and after Dicer knock-down in a MCF-7 cell line. The analysis reported here uses only the cytoplasmic sample pair (GSM769509 and GSM769511). Since short RNA processing takes place in this compartment we expect to reduce the noise from the nucleus.

All the adapter-free reads were mapped against the human genome (NCBI36.50 Release of July 2008) using segemehl (Hoffmann et al., 2009): we activated the poly-A clipping, required small RNAs to map with an accuracy of at least $90 \%$ and selected the "best scoring hit strategy". With these settings we mapped 8,743,377 of $15,493,265$ reads $(56 \%)$ of the control sample and $5,471,242$ of $9,237,490$ reads $(59 \%)$ of the Dicer knockdown sample. The resulting sam files were converted to bam format, using samtools (Li et al., 2009) and subsequently translated to bigWig files using a custom perl script. The read density at each position in the bigWig files was normalized by the number of multiple hits of each read and the absolute number of mapped reads of each experiment (RPM) in order to make the two experiments comparable. We provide custom tracks for the UCSC Genome Browser (Kent et al., 2002) to make the mapping results publicly available.

\subsection{Expressed Sites and Annotation}

In order to identify previously un-annotated loci with small RNA expression we created sorted bed files and then used blockbuster (Langenberger et al., 2009) with default parameters to identify regions showing accumulations of at least 50 reads in at least one of control or Dicer knock-down data. We used mergeBed from BEDtools (Quinlan and Hall, 2010) to obtain the final 
list of expressed regions of interest (1,946 for control and 1,798 for the knock-down set), which we call "sites" from now on.

We downloaded the latest annotations from different sources (1523 microRNA loci from miRBase v18 (Griffiths-Jones, 2004); 631 tRNA loci from gtRNAdb (Chan and Lowe, 2009); 402 snoRNA loci as well as 4528 other RNAs from UCSC annotation (Karolchik et al., 2004)). This combined annotation track comprising 7,084 annotated ncRNA loci was compared with with our list of sites using intersectBed (Quinlan and Hall, 2010).

Furthermore, all reads were overlapped with the UCSC repeat masker track (Jurka et al., 2000) and as soon as one read was mapped to a repeat associated region, all multiple hits of it were flagged with the type of repeat. If more than $50 \%$ of the expression of one site is caused by reads which are flagged as repeat associated, the whole site was flagged accordingly. In order to remove low-complexity sequences, which have a high probability of being random matches in short read data, we discarded all sites with a Shannon entropy of less than 1.6 bit.

\subsection{Expression Levels}

The expression level of each site, expressed in reads per kilobase of locus per million mapped reads (RPKM) was computed using the UCSC tool bigWigAverageOverBed (Kent et al., 2002). From these values we derived, for each site, the $\log _{2}$-fold change $\lambda$ between the Dicer knock-down sample and the RPKM of the control sample. All sites with $\lambda<0$ are interpreted as Dicer processed. All sites, together with their annotations, their expression values, their $\lambda$ and a link to the UCSC Genome Browser can be found at the supplement page http://www.bioinf.uni-leipzig.de/ supplements/12-005.

\subsection{Processing Pattern}

Cleavage of a nearly double-stranded RNA by Dicer leads to a characteristic 2 nt overhang at the 3'end, see e.g. $(\mathrm{Ji}, 2008)$. In order to assess how important the thermodynamic stability of the precursor structure is for processing, we computed for a pair of putative single-stranded cleavage products, the following stability measure: RNAcofold (Bernhart et al., 2006) is used to compute the energy of the duplex with the constraint that the joint structure exhibits the 2 nt overhang at the 3 'ends. Then the inner part of both sequences is shuffled 100 times so that the dinucleotide composition is preserved, while the terminal base pairs and overhanging
Table 1: Fraction of Dicer processed sites among the annotated ncRNAs.

\begin{tabular}{|c|c|c|c|c|}
\hline \multicolumn{5}{|c|}{ Dicer processed } \\
\hline type & yes & no & all & processed \\
\hline miRNA & 255 & 10 & 265 & $96.2 \%$ \\
\hline tRNA & 32 & 376 & 408 & $7.9 \%$ \\
\hline H/ACA snoRNA & 8 & 4 & 12 & $66.0 \%$ \\
\hline C/D snoRNA & 0 & 53 & 53 & $0.0 \%$ \\
\hline misc RNA & 3 & 2 & 5 & $60.0 \%$ \\
\hline snRNA & 2 & 92 & 94 & $2.1 \%$ \\
\hline scRNA & 10 & 90 & 100 & $10.0 \%$ \\
\hline rRNA & 28 & 254 & 282 & $9.9 \%$ \\
\hline
\end{tabular}

nucleotides were left untouched. The resulting $z$-score of the co-folding energies is recorded. For each site we considered the two consecutive tags with the largest expression as candidates for Dicer processing.

In order to assess the overall similarity of a site with canonical microRNAs we use RNAmicro (Hertel and Stadler, 2006). This tool evaluates structural features as well as the pattern sequence conservation. We retrieved alignments of all sites with 20nt flanking sequence on both sides from the 8 way-multiZ alignmemnt (human, chimp, orangutan, rhesus macaque, marmoset, mouse, opossum, platypus) (Blankenberg et al., 2011). We extracted sequences from 8 way-multiZ file, re-alignes them using clustalw (Larkin et al., 2007) and used it to run RNAmicro. Then, the RNAmicro decision value (decV) was used to rate the sites, if they microRNA-like structures and conservations.

Dicer is well known to generate products in the narrow length range 21-28 nt, see e.g. (Starega-Roslan et al., 2011). We therefore recorded the distribution of read lengths for each locus. In addition, we determined the lengths of blocks of reads blockbuster (Langenberger et al., 2009) with default parameters. Read blocks summarize groups of reads that overlap nearly perfectly, hence its lengths is typically larger than that of individual reads.

\section{Results}

\subsection{Identification of Dicer-dependent small RNAs}

The Dicer knock-down (GSM769509) and control (GSM769511) datasets (Friedländer et al., 2012) together identify 2,115 expressed sites. Of these, 1,048 overlap with the 7,084 annotated ncRNAs and 1,067 remain unannotated. After filtering out the low-complexity sites, we retain 1,002 annotated and 539 unknown sites for further analysis. 



Figure 1: Summary of expression changes of small, microRNA-sized RNAs in response to a knock-down of Dicer. The entire dataset is shown in grey, specific groups are highlighted as black dots. (a) Almost all annotated microRNAs are down-regulated, i.e., exhibit $\log _{2}$-fold changes $\lambda<0$. (b) Only a few tRNAs are downregulated. (c) None of the sdRNAs derived from box C/D snoRNAs is depeleted in response to the Dicer knock-down, while (d) the majority of the (small number of) sdRNAs derived from box H/ACA snoRNAs is Dicer dependent. (e) The small RNAs originating from Y RNAs and almost all Y RNA derived loci are not downregulated in response to Dicer knock down. (f) Mitochondrial transcripts and/or NUMTs are also a prolific source of small RNAs. These are independent of Dicer processing. Among repetitive elements, a substantial fraction of $(\mathrm{g})$ expressed SINEs and (h) expressed LINEs shows Dicer dependent processing.



Fig. 1 summarizes the response of the small RNA sites to Dicer knockdown. The $\log _{2}$-fold change $\lambda$ exhibits the expected bi-modal distribution separating in particular microRNAs from other small RNA products. Consistent with the original analysis of these datasets (Friedländer et al., 2012), microRNAs are strongly reduced upon reduction of Dicer activity. A closer inspection, however, shows a more differentiated picture.

On the one hand, a small subgroup of microRNAs does not respond to the knockdown of Dicer. On the other hand, a sizable number of unannotated sites (some of which might constitute previously undescribed microRNAs) are associated with well-known structured RNAs exhibiting large negative values of $\lambda$, see Table 1 .

A substantial fraction of sites expressing small RNAs are annotated repetitive elements, Table 2. Disregarding a moderate number of simple repeats and low complexity regions, which cannot be unambiguously distinguished 
Table 2: Fraction of Dicer processed sites among the NUMTs and repeat associated regions.

\begin{tabular}{lccrr}
\hline \multicolumn{4}{c}{ Dicer processed } & \\
type & yes & no & all & processed \\
\hline NUMT / chrM & 1 & 66 & 67 & $1.5 \%$ \\
SINE & 126 & 427 & 553 & $22.8 \%$ \\
LINE & 141 & 368 & 509 & $27.7 \%$ \\
LTR & 81 & 306 & 387 & $20.9 \%$ \\
DNA & 27 & 106 & 133 & $20.3 \%$ \\
Simple repeat & 18 & 66 & 84 & $21.4 \%$ \\
Low complexity & 15 & 15 & 30 & $50.0 \%$ \\
Other & 1 & 13 & 14 & $7.1 \%$ \\
Satellite & 1 & 2 & 3 & $33.3 \%$ \\
RNA & 1 & 2 & 3 & $33.3 \%$ \\
tRNA & 0 & 2 & 2 & $0.0 \%$ \\
\hline
\end{tabular}

from artefacts without further experimental evidence, we observe that about one fifth of repeat-associated small RNAs react to Dicer. This is not unexpected, as repetitive elements are one of the documented sources of novel microRNAs. Smalheiser and Torvik (2005), for instance showed that a subset of conventional mammalian microRNAs is derived from LINE-2 transposable elements. A family of miRNAs deriving from miniature invertedrepeat transposable elements (MITES) has been characterized by (Piriyapongsa and Jordan, 2007). A recent comprehensive analysis of microRNAs originating from transposable elements can be found in (Borchert et al., 2011), see also (Yuan et al., 2011).

\subsection{Characterization of Dicer-processed sites}

Dicer-processed small RNAs typically derive from helical regions that are significantly more stable than the precursor secondary structures of Dicer-independent small RNAs. Fig. 2a shows that in particular putative precursor structures that give rise to the typical processing patterns with 2 nt overhangs are substantially stabilized Dicer-responsive small RNAs.

Canonical microRNAs also exhibit a characteristic pattern of sequence conservation that can help to distinguish them from other, similar, sources of small RNAs and from hairpin-like structures that are not processed into small RNAs, see e.g. (Lai et al., 2003). RNAmicro (Hertel and Stadler, 2006) implements such a classifier based on a Support Vector Machine taking only a small number of structural and conservation based descriptors as input. Only sites that form hairpin structures can be scored by RNAmicro's SVM. We have previously used RNAmi cro to distinguish microRNA-like from snoRNAlike small RNA sites (Langenberger et al., 2011). Fig. 2b
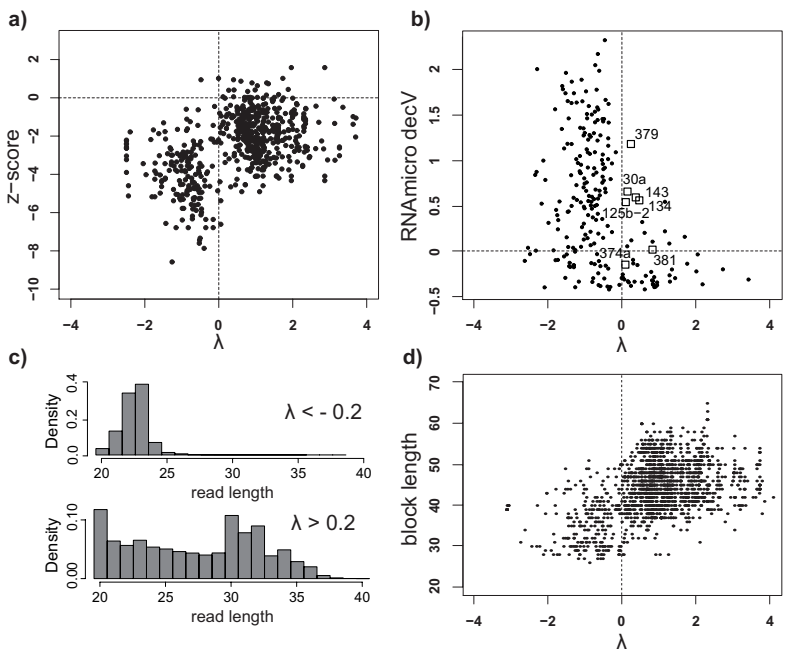

Figure 2: Correlation of Dicer-response $\lambda$ with sequence-derived descriptors on the entire data set: (a) Free energy $z$-scores of constrained duplex structures with the 2-nt overhangs conforming to the canonical Dicer processing pattern. (b) Decision value of RNAmicro, a SVMbased machine learning tool trained to recognized canonical microRNAs. Its decision value combines the stability of the hairpin structure with patterns of sequence conservation but is agnostic about the location of the small RNA products. Only sites that form a hairpin structure can be scored by this method. (c) Reads with arising from Dicer processed regions, i.e. those with small values of $\lambda$, have a length within the size range between 20 and $25 \mathrm{nt}$ typical for microRNAs. The length distribution of reads without evidence for Dicer-processing is much broader and most reads fall outside the expected size range. (d) The reads orginating from Dicer-responsive RNA form shorter, more coherent read blocks.

shows that the RNAmicro decision value is also correlated with $\lambda$. With few exceptions, large decision values are limited to Dicer responsive sites.

Fig 2c summarizes the distribution of read lengths. As expected, nearly all reads arising from sites with $\lambda<-0.2$ have a lengths between 20 and 25nt, consistent with Dicer processing (Starega-Roslan et al., 2011). In contrast, short reads from sites with $\lambda>0.2$, i.e., those that are clearly not resulting from Dicer cleavage, are typically longer and show a flat distribution. We also observe a difference in the length of read blocks as determined by blockbuster (Langenberger et al., 2009). Sites with $\lambda<0$ have on average much shorter block sizes, often consisting only of a single block of microRNAs, Fig. 2d. Since the start and end position of mature microRNAs can vary by a couple of nucleotides (Ebhardt et al., 2010) such that overlapping microRNAs read blocks have a length of around $30 \mathrm{nt}$. 


\subsection{Structured regions processed by Dicer indicate po- tential microRNA candidates}

The data set used here has been generated specifically for the purpose of detecting novel microRNAs (Friedländer et al., 2012). Among the un-annotated, nonrepetitive sites with $\lambda<0$ six additional structured regions (Figure 3 ) were found. One of them (Figure 3a) is located in an intergenic region Figure $3 \mathrm{a}$, is located in intergenic region far away from any annotation. Three of four intronic sequences (Figure 3d-f) fold into hairpins and the short reads map to the stem positions expected for mirtrons (Okamura et al., 2007; Ruby et al., 2007). The 3' end of a candidate located in a SYT12-intron is determined by the splice acceptor (Figure 3b). Given its stable hairpin structure these findings suggest that it belongs to the recently described class of "semi-mirtrons" that require both splicing and exosome-mediated trimming for maturation (Flynt et al., 2010; Chong et al., 2010). The structure and the positions of mapped reads of the remaining candidate (Figure 3c) do not conform to a typical microRNA. Nevertheless, the ten-fold reduction of the read coverage in the Dicer knockdown experiment indicates Dicer-processing. Since these reads perfectly but not uniquely map to the intronic region, this candidate is of particular interest for further analysis.

\subsection{Dicer-processed non-microRNAs}

Surprisingly, there is a large number of well-known structured non-coding RNAs from which Dicer-sensitive small RNAs are produced.

A prominent example are the vault RNAs. The largest response is observed for vtRNA2-1 with $\lambda=-2.12$. This locus was originally classified as hsa-mir-886 but lateron recognized as a polymerase-III transcript (Canella et al., 2010) and vault RNA paralog (Nandy et al., 2009; Stadler et al., 2009). The other three vault RNA loci also give rise to short RNAs (Persson et al., 2009) and respond negatively to the Dicer depletion: $\lambda($ vtRNA1-1 $)=$ $-0.14, \lambda($ vtRNA1-2) $=-0.76$. The vtRNA1-3 locus is not sufficiently expressed.

The snaR ncRNAs (Parrott and Mathews, 2007) are pol-III transcripts that emerged in the ancestor of the African Great Apes from an Alu-derived precursor (Raha et al., 2010; Parrott et al., 2011). Fig. 4 shows that microRNA-like small RNAs are processed from the lower end of the stem-loop structure, which resembles a canonical pre-microRNA hairpin except for its length of more than 100nt. The snaR-derived small RNAs show the typical 2 nt 3' overhangs. Their expression depends very strongly on the Dicer concentration.
The situation is more complex for tRNAs and snoRNAs. While many of them give rise to small RNA products, the majority is not influenced by the Dicer knockdown. A small subset of tRNAs, on the other hand is clearly subject to Dicer processing. These include in particular tRNA-Gln-CTG with $\lambda=-2.05$ as noted already previously by Cole et al. (2009). Other tRNAs with a clear Dicer signature are tRNA-Asn-GTT $(\lambda=-1.47)$, tRNA-Asn-ATT $(\lambda=-0.83)$, tRNA-Ala-CGC $(\lambda=$ -1.28), tRNA-Ile-TAT ( $\lambda=-1.19)$, tRNA-Glu-TTC $(\lambda=-0.79)$. None of the four mirbase "microRNAs" that are derived from tRNAs (mir-1274/tRNA-Lys, mir1280/tRNA-Leu, mir-720/tRNA-Thr, mir-1308/tRNAGly) are expressed at sufficiently high levels to estimate $\lambda$.

Small nucleolar RNAs can share several characteristics with microRNAs, including similar components in their processing, see (Scott and Ono, 2011) for a recent review. The structural similarities between H/ACA snoRNAs and microRNAs are most obvious and have been noticed in several computational studies. Scott et al. (2009), for instance, report twenty miRNA precursors that show significant similarity to H/ACA snoRNAs; of these miR-151, miR-605, mir-664 = SNORA36B, miR215 , and miR-140 even bind to dyskerin, a component of the H/ACA snoRNP. On the other hand, Dicer processing has been demonstrated previously for SNORA45 (Ender et al., 2008). Consistently, we find $\lambda($ SNORA45) $=$ -1.55 . Of the $12 \mathrm{H} / \mathrm{ACA}$ snoRNAs with sufficient expression 8 have $\lambda<0$ (Table 3 ), indicating that short reads from $\mathrm{H} / \mathrm{ACA}$ snoRNAs are typically a product of Dicer processing. Interestingly, two H/ACA snoRNAs were classified as novel microRNAs by mirdeep2 (Friedländer et al., 2012): SNORA36A $(\lambda=-1.33)$ and SNORA33 $\lambda=0.15$. We emphasize, however, that only a small minority of H/ACA snoRNAs leads to abundant processing products. In addition these small RNAs are independent of Drosha (Ender et al., 2008; Taft et al., 2009; Brameier et al., 2011), and in some cases Drosha even inhibits sdRNA formation (Taft et al., 2009), emphasizing that the snoRNAs and (canonical) microRNAs are in general clearly distinguished entities.

A quite different picture emerges for box C/D snoRNAs. Although small RNAs are abundantly produced from box C/D snoRNAs in our data set, Tab. 1, there is no indication that any of them is a Dicer substrate. The box C/D snoRNAs that are discussed as possibly microRNA-like in (Langenberger et al., 2011) show only marginal expression levels and no indication for Dicer processing. On the other hand, of the five microRNAs that resemble box C/D snoRNAs (having $\mathrm{C}$ and $\mathrm{D}$ boxes 

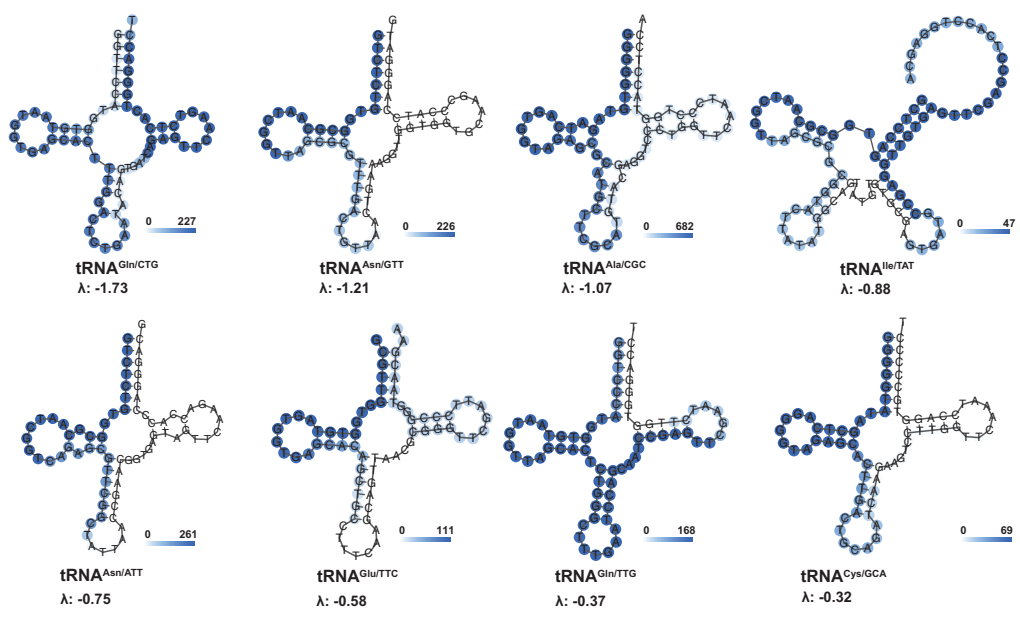

Figure 5: Several tRNAs are processed into small RNAs by Dicer. The processing patterns shown some similarities, in particular a tendency to have large short read coverage on the 3' side of the tRNA clover leaf. With the exception of tRNA-Ile-TAT the small RNAs are derived from within the mature tRNA.

Table 3: Box H/ACA snoRNAs processed by Dicer. SNORA36B (Ender et al., 2008) (also annotated as mir-664) does not reach a sufficent expression level in MCF-7 cells.

\begin{tabular}{lclc}
\hline snoRNA & $\lambda$ & snoRNA & $\lambda$ \\
\hline SNORA45 & -1.55 & SNORA36B & $*$ \\
\hline SNORA51 & -2.42 & SNORA46 & -1.00 \\
SNORA36A & -1.33 & SNORA56 & -0.93 \\
SNORA17 & -1.19 & SNORA7B & -0.66 \\
SCARNA3 & -1.13 & SNORA7A & -0.28 \\
\hline
\end{tabular}

in close proximity in the precursor and binding to fibrillarin) (Ono et al., 2011), four are Dicer substrates (miR-27b $\lambda=-0.90$, miR-16-1 $\lambda=-0.40$, mir-28 $\lambda=-0.95$, and let-7g $\lambda=-1.16$ ) and the fifth (mir31 ) is not sufficiently expressed in MCF-7 cells. It appears, thus, that Dicer-processing clearly distinguished between bona fide microRNAs and small RNAs derived from box C/D snoRNAs.

Y RNAs are small pol-III transcripts that originate from RNA component of the Ro RNP particle and have a role in DNA replication (Christov et al., 2006). The four paralogous human Y RNAs form a cluster on Chr.7(148M) (Mosig et al., 2007; Perreault et al., 2007). The canonical loci show no evidence of Dicer processing hY3 $\lambda=0.12$, hY4 $\lambda=1.25$, hY1 $\lambda=1.70$, hY5 $\lambda=1.74$. We note that fragments from hY5 have also been annotated as mir-1975.

In addition to the canonical Y RNA cluster, however, there are more than a thousand Y RNA pseudogenes scattered across the genome (Perreault et al., 2007). The deep sequencing data shows that several of these loci form a source of short reads. A few of the Y4-derived loci sites have negative values of $\lambda$. We note, however, these have relatively low expression levels and might be confounded by mapping artefacts. In total, 11 sites that are derived from Y RNA sequences are classified as microRNAs by RNAmicro, six of which have moderate negative values of $\lambda$.

\subsection{MicroRNA not processed by Dicer}

The best-studied microRNA that is not processed by Dicer is mir-451. Unfortunately this site is not significantly expressed in MCF-7 cells, so that we cannot use it as a control. There are ten additional microRNAs with $\lambda>0$. Six of them (mir-30a, mir-143, mir-374a, mir379, mir-381, and mir-134) derive from precursor hairpins that are recognized by RNAmicro. Two of these, mir-30a and mir-374a, exhibit exceptionally high levels of expression and feature short RNAs derived from both sides of the precursor stem, Figure 7 . We suspect that they are exceptionally good substrates for Dicer so that their maturation is least affected by Dicer concentrations. The evolutionarily ancient mir-125b-2 also exhibits both a canonical read pattern and a canonical pattern of sequence conservation. Nevertheless, it shows no reaction to Dicer knockdown, $\lambda=0.03$.

For mir-143, mir-381, mir-134, mir-4417, and mir4516 no mir* reads were detectable. Mir-4417 is present in monkeys only (Supplemental Material), and no homologs are detectable for hsa-mir-4516, precluding the analysis of patterns of sequence conservation for these two microRNAs.

The entire precursor hairpin of mir-3676 is covered by small RNA sequences. A closer inspection shows, however, that mir-3676 coincides with tRNAThr-AGT and is thus clearly an erroneous annotation. The mis-annotated 


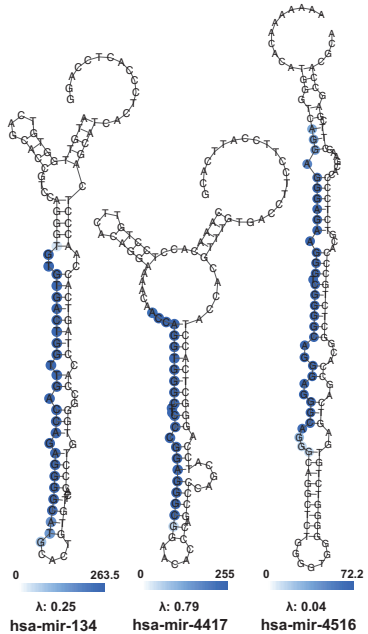

Figure 7: MicroRNAs that are not processed by Dicer.

"mir-3195", furthermore, corresponds to a GC-rich lowcomplexity region located with the first exon of the TAF4 gene.

The sequence of mir-663a is very GU-rich and does not meet our exclusion criterion for low-complexity sequences. We retained it in our data set because it is well document as an important tumor suppressor (Pan et al., 2010; Yi et al., 2012). In contrast to canonical microRNAs, its primary sequence is quite poorly conserved although it can be found throughout the major eutherian groups. Its read pattern also strongly deviates from the expectation for microRNAs. Similar to mir-451, the precursor is covered with a background of short reads, as also seen in the cummulative read patterns provided by the MicroRNA Registry, albeit there is dominating, most frequently produced "mature microRNAs".

\section{Discussion}

A rapidly expanding zoo of diverse small RNA species has emerged following the discovery of RNA interference (Fire et al., 1998) and microRNAs (Lee et al., 1993) almost two decades ago. With the rapid increase of high throughput sequencing data the boundaries between the different subdivisions of small RNAs have become more and more blurry.

Here we have focussed on the generation of small RNAs from their double-stranded precursors. Making use of a publicly available dataset (Friedländer et al., 2012) we find, consistent with the well-established knowledge, that the overwhelming majority of miRBase microRNAs is processed by Dicer. There are, however, several notable exceptions. Cole et al. (2009) argue that
Dicer knockdown with siRNAs for a short period of time sometimes does not result in a significant change in the miRNA steady state level due to slow microRNA turnover. At least some of the Dicer-unresponsive miRNAs, however, exhibit unusual structural features and/or read patterns that deviate substantially from canonical microRNAs. While $\lambda>0$ in itself is course not sufficient proof for Dicer-independence, it is at least a strong indication and helps to identify candidates for further analysis.

Dicer-processing is not limited to microRNAs. Several polymerase-III transcripts are prolific Dicer substrates, including human vault RNAs, the great ape specific snaRs, and a small set of about a dozen tRNAs. While the vault RNAs products function like microRNAs, small RNAs derived from tRNA-Gln-CTG do not function in this way: they do not associate with argonaut presumably due to the fact that these small RNAs are just too small (Cole et al., 2009). Despite their similarity with vault RNAs, including a secondary structure with a long terminal stem, there is no evidence that the abundant small RNAs deriving from Y RNAs are produced by Dicer cleavage. Both main classes of small nucleolar RNAs are sources of abundant small RNAs. While all of the highly expressed box C/D snoRNAs are processed independently of Dicer, the situation is different for H/ACA snoRNAs. Most box H/ACA snoRNAs are a source of small RNAs, but in most cases the expression levels are small, at least in the invesitaged MCF-7 libraries. Among the highly expressed ones, however, the majority clearly is a Dicer substrate.

In summary, there does not seem to be a clear separation between between processing pathways resulting in 
small RNAs. Instead, the picture of an intricate network of interleaved alternatives emerges, in which the individual processing steps can be freely combined. As a consequence, it appears that a particular sequence of processing steps is neither a sufficient nor a necessary condition for a particular role. Small RNA sequencing data such as the ones analyzed here reveal only the end points of a likely more complex processing cascade. Longer potential intermediates, such as pre-microRNA hairpins or the parts of tRNAs resulting from stress-related cleavage in the anticodon loop (Jöchl et al., 2008; Thompson et al., 2008) are invisible here. It will be an interesting topic for future research to investigate if and how the generation of small RNAs is linked to other RNA processing mechanisms.

\section{Acknowledgments}

This work is supported in part by the Deutsche Forschungsgemeinschaft (SPP-1258 "Sensory and Regulatory RNAs in Prokaryotes": STA 850/7-2 to PFS). LIFE - Leipzig Research Center for Civilization Diseases, Universität Leipzig is funded by means of the European Social Fund and the Free State of Saxony.

\section{Conflict of interest}

The authors declare no conflict of interest.

\section{References}

Babiarz JE, Ruby JG, Wang Y, Bartel DP, Blelloch R, 2008. Mouse ES cells express endogenous shRNAs, siRNAs, and other Microprocessor-independent, Dicer-dependent small RNAs. Genes Dev 22:2773-2785.

Bernhart SH, Tafer H, Mückstein U, Flamm C, Stadler PF, Hofacker IL, 2006. Partition function and base pairing probabilities of RNA heterodimers. Algorithms Mol Biol 1:3.

Blankenberg D, Taylor J, Nekrutenko A, Team TG, 2011. Making whole genome multiple alignments usable for biologists. Bioinformatics 27:2426-2428.

Borchert GM, Holton NW, Williams JD, Hernan WL, Bishop IP, Dembosky JA, Elste JE, Gregoire NS, Kim JA, Koehler WW, Lengerich JC, Medema AA, Nguyen MA, Ower GD, Rarick MA, Strong BN Tardi NJ, Tasker NM, Wozniak DJ, Gatto C, Larson ED, 2011. Comprehensive analysis of microRNA genomic loci identifies pervasive repetitive-element origins. Mob Genet Elements 1:8-17.

Brameier M, Herwig A, Reinhardt R, Walter L, Gruber J, 2011. Human box C/D snoRNAs with miRNA like functions: expanding the range of regulatory RNAs. Nucleic Acids Res 39:675-686.

Canella D, Praz V, Reina JH, Cousin P, Hernandez N, 2010. Defining the RNA polymerase III transcriptome: Genome-wide localization of the RNA polymerase III transcription machinery in human cells. Genome Res 20:710-721.

Chan P, Lowe T, 2009. GtRNAdb: a database of transfer RNA genes detected in genomic sequence. Nucleic Acids Res 37:D93-D97.
Cheloufi S, Dos Santos CO, Chong MM, Hannon GJ, 2010. A dicerindependent miRNA biogenesis pathway that requires Ago catalysis. Nature 465:584-589.

Chong MMW, Zhang G, Cheloufi S, Neubert TA, Hannon GJ, Littman DR, 2010. Canonical and alternate functions of the microRNA biogenesis machinery. Genes Dev 24:1951-1960.

Christov CP, Gardiner TJ, Szüts D, Krude T, 2006. Functional requirement of noncoding Y RNAs for human chromosomal DNA replication. Mol Cell Biol 26:6993-7004.

Cifuentes D, Xue H, Taylor DW, Patnode H, Mishima Y, Cheloufi S, Ma E, Mane S, Hannon GJ, Lawson ND, Wolfe SA, Giraldez AJ, 2010. A novel miRNA processing pathway independent of Dicer requires Argonaute2 catalytic activity. Science 328:1694-1698.

Cole C, Sobala A, Lu C, Thatcher SR, Bowman A, Brown JW, Green PJ, Barton GJ, Hutvagner G, 2009. Filtering of deep sequencing data reveals the existence of abundant Dicer-dependent small RNAs derived from tRNAs. RNA 15:2147-2160.

Ebhardt HA, Fedynak A, Fahlman RP, 2010. Naturally occurring variations in sequence length creates microRNA isoforms that differ in argonaute effector complex specificity. Silence 1:12.

Edgar R, Domrachev M, Lash A, 2002. Gene expression omnibus: Ncbi gene expression and hybridization array data repository. $\mathrm{Nu}-$ cleic Acids Res 30:207-210.

Ender C, Krek A, Friedländer MR, Beitzinger M, Weinmann L, Chen W, Pfeffer S, Rajewsky N, Meister G, 2008. A human snoRNA with microRNA-like functions. Mol Cell 32:519-528.

Findeiß S, Langenberger D, Stadler PF, Hoffmann S, 2011. Traces of post-transcriptional RNA modifications in deep sequencing data. Biol Chem 392:305-313.

Fire A, Xu S, Montgomery MK, Kostas SA, Driver SE, Mello CC, 1998. Potent and specific genetic interference by double-stranded RNA in Caenorhabditis elegans. Nature 391:806-811.

Flynt AS, Greimann JC, Chung WJ, Lima CD, Lai EC, 2010. MicroRNA biogenesis via splicing and exosome-mediated trimming in Drosophila. Mol Cell 38:900-907.

Friedländer MR, Mackowiak SD, Li N, Chen W, Rajewsky N, 2012. miRDeep2 accurately identifies known and hundreds of novel microRNA genes in seven animal clades. Nucleic Acids Res 40:37-52.

Gregory RI, Yan KP, Amuthan G, Chendrimada T, Doratotaj B, Cooch $\mathrm{N}$, Shiekhattar R, 2004. The Microprocessor complex mediates the genesis of microRNAs. Nature 432:235-240.

Griffiths-Jones S, 2004. The microRNA registry. Nucleic Acids Res 32:D109-D111.

Haussecker D, Huang Y, Lau A, Parameswaran P, Fire AZ, Kay MA, 2010. Human tRNA-derived small RNAs in the global regulation of RNA silencing. RNA 16:673-695.

Hertel J, Stadler PF, 2006. Hairpins in a haystack: Recognizing microRNA precursors in comparative genomics data. Bioinformatics 22:e197-e202.

Hoffmann S, Otto C, Kurtz S, Sharma CM, Khaitovich P, Vogel J, Stadler PF, Hackermüller J, 2009. Fast mapping of short sequences with mismatches, insertions and deletions using index structures. PLoS Comput Biol 5:e1000502.

Ji X, 2008. The mechanism of rnase III action: how dicer dices. Curr Top Microbiol Immunol 320:99-116.

Jöchl C, Rederstorff M, Hertel J, Stadler PF, Hofacker IL, Schrettl M, Haas H, Hüttenhofer A, 2008. Small ncRNA transcriptome analysis from Aspergillus fumigatus suggests a novel mechanism for regulation of protein-synthesis. Nucleic Acids Res 36:2677-2689.

Jurka J, et al., 2000. Repbase update: a database and an electronic journal of repetitive elements. Trends Genet 16:418-420.

Karolchik D, Hinrichs A, Furey T, Roskin K, Sugnet C, Haussler D, Kent W, 2004. The UCSC table browser data retrieval tool. Nucleic Acids Res 32:D493-D496. 
Kawaji H, Nakamura M, Takahashi1 Y, Sandelin A, Katayama S, email Fukuda S, Daub C, Kai C, Jun Kawai J, Yasuda J, Carninci P, Hayashizaki Y, 2008. Hidden layers of human small RNAs. BMC Genomics 9:157.

Kent WJ, Sugnet CW, Furey TS, Roskin KM, Pringle TH, Zahler AM, Haussler D, et al., 2002. The human genome browser at ucsc. Genome Res 12:996-1006.

Lai EC, Tomancak P, Williams RW, Rubin GM, 2003. Computational identification of drosophila microRNA genes. Genome Biol 4:R42.

Langenberger D, Bartschat S, Hertel J, Hoffmann S, Tafer H, Stadler PF, 2011. Microrna or not microrna? In: de Souza ON, Telles GP, Palakal MJ, editors, Advances in Bioinformatics and Computational Biology, 6th Brazilian Symposium on Bioinformatics, BSB 2011, vol. 6832 of Lecture Notes in Computer Science, (pp. 1-9). Berlin, Heidelberg: Springer.

Langenberger D, Bermudez-Santana C, Hertel J, Hoffmann S, Khaitovich P, Stadler PF, 2009. Evidence for human microrna-offset rnas in small rna sequencing data. Bioinformatics 25:2298-2301.

Langenberger D, Bermudez-Santana C, Stadler PF, Hoffmann S, 2010. Identification and classification of small RNAs in transcriptome sequence data. Pac Symp Biocomput 15:80-87.

Larkin MA, Blackshields G, Brown NP, Chenna R, McGettigan PA, McWilliam H, Valentin F, Wallace IM, Wilm A, Lopez R, Thompson J, Gibson TJ, Higgins DG, 2007. Clustal w and clustal x version 2.0. Bioinformatics 23:2947-2948.

Lee RC, Feinbaum RL, Ambros V, 1993. The C. elegans heterochronic gene lin-4 encodes small RNAs with antisense complementarity to lin-14. Cell 75:843-854.

Lee YS, Shibata Y, Malhotra A, Dutta A, 2009. A novel class of small RNAs: tRNA-derived RNA fragments (tRFs). Genes Dev 23:26392649.

Li H, Handsaker B, Wysoker A, Fennell T, Ruan J, Homer N, Marth G, Abecasis G, Durbin R, et al., 2009. The sequence alignment/map format and samtools. Bioinformatics 25:2078-2079.

Lund E, Güttinger S, Calado A, Dahlberg J, Kutay U, 2004. Nuclear export of microRNA precursors. Science 303:95-98.

Meiri E, Levy A, Benjamin H, Ben-David M, Cohen L, Dov A, Dromi N, Elyakim E, Yerushalmi N, Zion O, Lithwick-Yanai G, Sitbon E, 2010. Discovery of microRNAs and other small RNAs in solid tumors. Nucleic Acids Res 38:6234-6246.

Mosig A, Guofeng M, Stadler BMR, Stadler PF, 2007. Evolution of the vertebrate Y RNA cluster. Th Biosci 126:9-14.

Murchison EP, Hannon GJ, 2004. miRNAs on the move: miRNA biogenesis and the RNAi machinery. Curr Opin Cell Biol 16:223-229.

Nandy C, Mrázek J, Stoiber H, Grässer FA, Hüttenhofer A, Polacek N, 2009. Epstein-Barr virus-induced expression of a novel human vault RNA. J Mol Biol 388:776-784.

Okamura K, Hagen JW, Duan H, Tyler DM, Lai EC, 2007. The mirtron pathway generates microRNA-class regulatory RNAs in Drosophila. Cell 130:89-100.

Ono M, Scott MS, Yamada K, Avolio F, Barton GJ, Lamond AI, 2011. Identification of human miRNA precursors that resemble box C/D snoRNAs. Nucleic Acids Res 39:3879-3891.

Pan J, Hu H, Zhou Z, Sun L, Peng L, Yu L, Sun L, Liu J, Yang Z, Ran Y, 2010. Tumor-suppressive mir-663 gene induces mitotic catastrophe growth arrest in human gastric cancer cells. Oncol Rep 24:105-112.

Parrott AM, Mathews MB, 2007. Novel rapidly evolving hominid RNAs bind nuclear factor 90 and display tissue-restricted distribution. Nucleic Acids Res 35:6249-6258.

Parrott AM, Tsai M, Batchu P, Ryan K, Ozer HL, Tian B, Mathews $\mathrm{MB}, 2011$. The evolution and expression of the snaR family of small non-coding RNAs. Nucleic Acids Res 39:1485-1500.

Perreault J, Perreault JP, Boire G, 2007. Ro-associated Y RNAs in metazoans: evolution and diversification. Mol Biol Evol 24:1678-
1689

Persson H, Kvist A, Vallon-Christersson J, Medstrand P, Borg A, Rovira C, 2009. The non-coding RNA of the multidrug resistancelinked vault particle encodes multiple regulatory small RNAs. Nat Cell Biol 11:1268-1271.

Piriyapongsa J, Jordan IK, 2007. A family of human microRNA genes from miniature inverted-repeat transposable elements. PLoS One 2:e203.

Quinlan A, Hall I, 2010. BEDTools: a flexible suite of utilities for comparing genomic features. Bioinformatics 26:841-842.

Raha D, Wang Z, Moqtaderi Z, Wu L, Zhong G, Gerstein M, Struhl K, Snyder M, 2010. Close association of RNA polymerase II and many transcription factors with Pol III genes. Proc Natl Acad Sci USA 107:3639-3644.

Ruby GJ, Jan CH, Bartell DP, 2007. Intronic microRNA precursors that bypass Drosha processing. Nature 48:83-86.

Scott MS, Avolio F, Ono M, Lamond AI, Barton GJ, 2009. Human miRNA precursors with box H/ACA snoRNA features. PLoS Comput Biol 5:e1000507.

Scott MS, Ono M, 2011. From snoRNA to miRNA: Dual function regulatory non-coding RNAs. Biochimie 93:1987-1992.

Smalheiser NR, Torvik VI, 2005. Mammalian microRNAs derived from genomic repeats. Trends Genet 21:322-326.

Sobala A, Hutvagner G, 2011. Transfer RNA-derived fragments: origins, processing, and functions. Wiley Interdiscip Rev RNA 2:853862.

Stadler PF, Chen JJL, Hackermüller J, Hoffmann S, Horn F, Khaitovich P, Kretzschmar AK, Mosig A, Prohaska SJ, Qi X, Schutt K, Ullmann K, 2009. Evolution of vault RNAs. Mol Biol Evol 26:1975-1991.

Starega-Roslan J, Krol J, Koscianska E, Kozlowski P, Szlachcic WJ, Sobczak K, Krzyzosiak WJ, 2011. Structural basis of microRNA length variety. Nucleic Acids Res 39:257-268.

Taft RJ, Glazov EA, Lassmann T, Hayashizaki Y, Carninci P, Mattick JS, 2009. Small RNAs derived from snoRNAs. RNA 15:1233-1240.

Thompson DM, Lu C, Green PJ, Parker R, 2008. tRNA cleavage is a conserved response to oxidative stress in eukaryotes. RNA 14:20952103.

Verhagen AP, Pruijn GJ, 2011. Are the Ro RNP-associated Y RNAs concealing microRNAs? Y RNA-derived miRNAs may be involved in autoimmunity. Bioessays 33:674-682.

Yang JS, Lai EC, 2011. Alternative miRNA biogenesis pathways and the interpretation of core miRNA pathway mutants. Mol Cell 43:892-903.

Yi C, Wang Q, Wang L, Huang Y, Li L, Liu L, Zhou X, Xie G, Kang T, Wang H, Zeng M, Ma J, Zeng Y, Yun JP, 2012. MiR-663, a microRNA targeting $\mathrm{p} 21$ (WAF1/CIP1), promotes the proliferation and tumorigenesis of nasopharyngeal carcinoma. Oncogene Doi: 10.1038/onc.2011.629.

Yuan Z, Sun XS, Liu H, Xie J, 2011. MicroRNA genes derived from repetitive elements and expanded by segmental duplication events in mammalian genomes. PLoS ONE 6:e17666. 




$\begin{array}{lr}0 & 110 \\ \lambda:-2.37\end{array}$

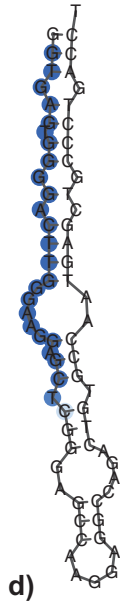

$\lambda:-1.93$

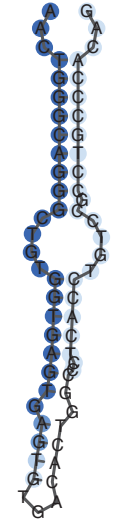

b)
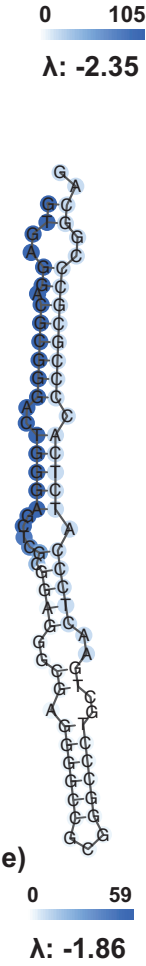

c)
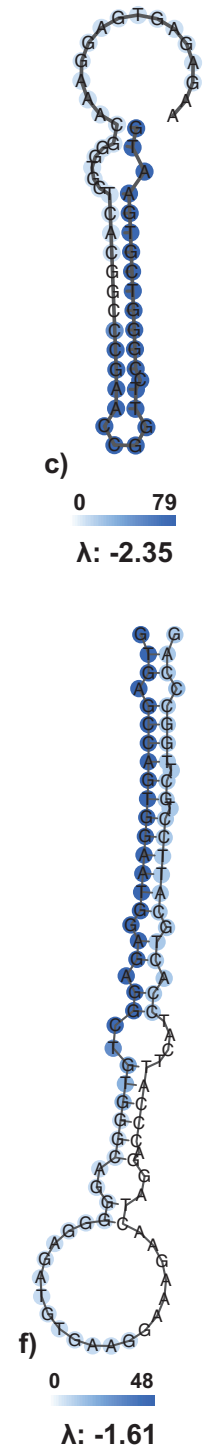

Figure 3: Six un-annotated non-repetitive loci that are processed by Dicer. (a) intergenic chr2:81,100,049-81,100,134(+); (b) a "semimirtron" in an intron of SYT12 chr11:66,569,729-66,569,790(+); (c) a source in an intron of SLC4A2 chr7:150,394,782-150,394,835(+); three mirtrons: (c) SLC4A2 chr7:150,394,782-150,394,835(+); (d) FLNA chrX:153,235,873-153,235,943(-); (e) MAP3K4 chr1:27,559,917-27,559,998(-); (f) TRIM28 chr19:63,753,464$63,753,555(+)$. The color scale represents the coverage on a logarithmic scale.

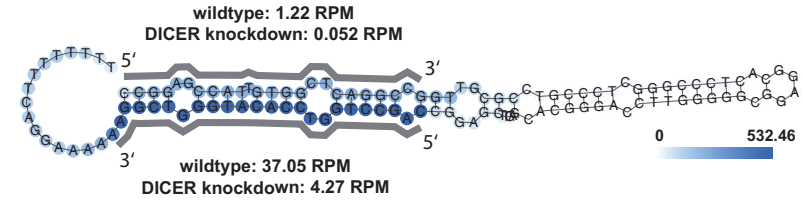

Figure 4: snaRs are processed by Dicer. Highlighted are the tags showing the highest expression.

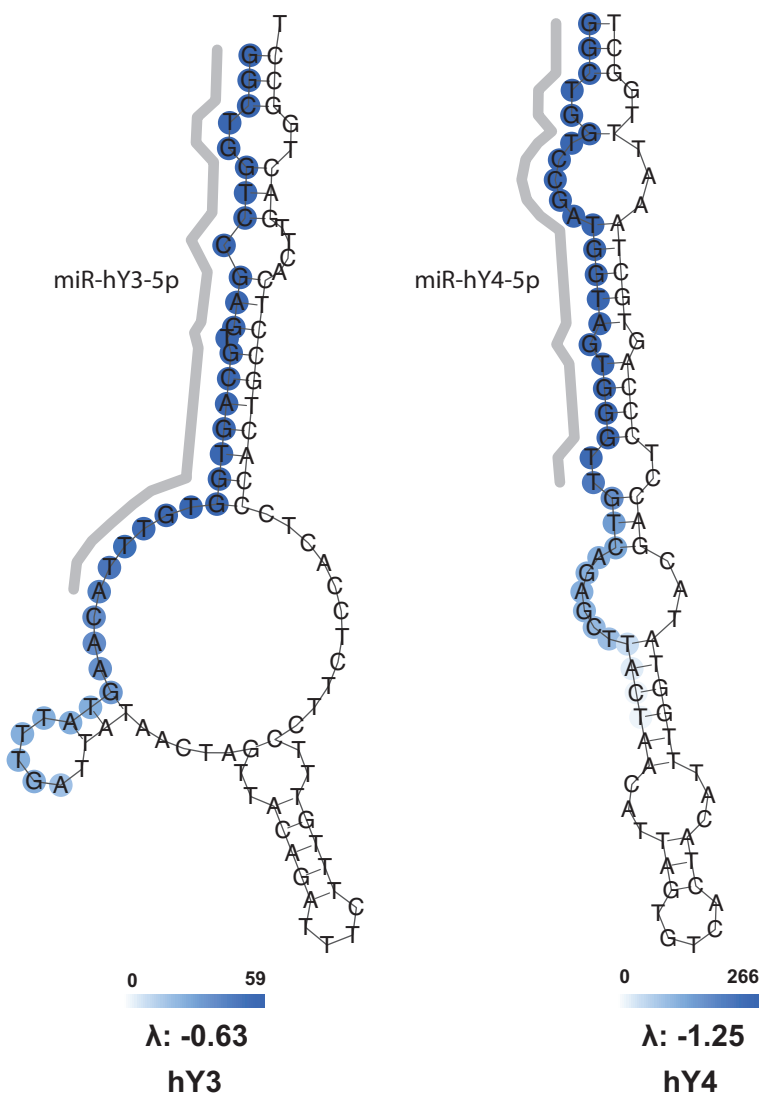

Figure 6: Small RNAs derived from Y RNAs. miR-hY3-5p and miRhY4-5p (Verhagen and Pruijn, 2011) are highlighted. 